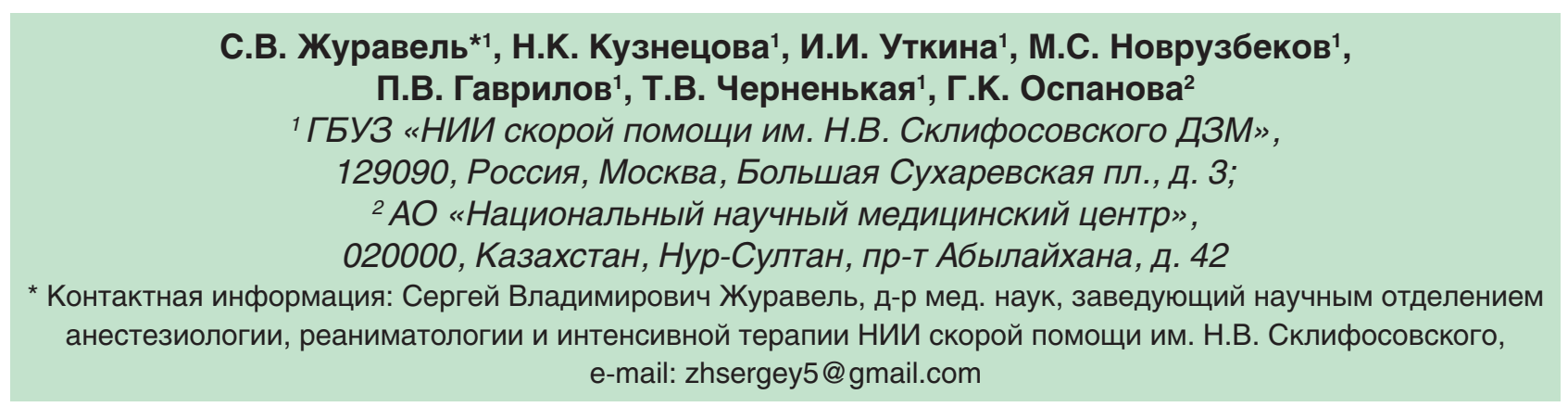

Введение. Развитие инвазивного кандидоза приводит к высокой слертности после трансплантации печени. Выбор эбббективного профилактического средства является важной задачей.

Цель исследования. Сравнение результатов пробилактики инвазивного кандидоза анидулабунгином и липидной бормой амфотерицина В у паииентов высокого риска в раннем послеопераиионном периоде после трансплантации печени.

Материал и методы. В исследование включили 80 паииентов, перенесших трансплантацию печени с бакторали риска развития инвазивного микоза. Пациенты бъли разделенъ на две группъ. В 1-й группе (n=40) с иелъю профилактики назначали анидулабунгин, во 2-й группе $(n=40)$ применяли липидную фборму алфотерицина В. Результаты. Самым распространенным видом Сапdida spр., выявленных у пациентов в нашем исследовании, ожидаемо являласъ Candida albicans, при этом ее доля составила значительно менъше половинъ- $31,2 \%$. Не бъло зарегистрировано прорыва грибковой инфекции и развития инвазивного микоза ни у одного пациента. В группе 2 статистически значимо чаще использовалась заместительная почечная терапия. В 2 случаях осуществленъ отмена липидного комплекса алфотерицина В и конверсия на эхинокандин в связи с появлением нежелательнъх явлений (озноб и высокая температура), связанных, на наш взгляд, с применяемыл лекарственныл средством. Выводы. 1. Для профилактики инвазивного микоза имеются абсолютные показания у пациентов после трансплантации печени с двумя и более фбакторами риска. 2. Анидулабунгин и липидная фборма алфотерицина В эбббективны для профилактики и предотвращения «прорыва» грибковой инфекиии. 3. Анидулабунгин имеет преимущество перед липидной бормой амботерицина В как более безопасное средство.

Ключевые слова: профилактика грибковой инфекции, трансплантация печени, анидулафунгин, липидная форма амфотерицина $B$ $\begin{array}{ll}\text { КонФликт интеРесов } & \text { Авторы заявляют об отсутствии конфлликта интересов } \\ \text { ФинансиРовАниЕ } & \text { Исследование проводилось без спонсорской поддержки }\end{array}$

Журавель С.В., Кузнецова Н.К., Уткина И.И., Новрузбеков М.С., Гаврилов П.В., Черненькая Т.В. и др. Сравнение результатов профилактики инвазивного кандидоза анидулафунгином и липидной формой амфотерицина $B$ у пациентов высокого риска в раннем послеоперационном периоде после трансплантации печени. Трансплантология. 2020;12(2):104-111. https://doi. org/10.23873/2074-0506-2020-12-2-104-111 


\title{
Comparing the results of invasive candidiasis prevention with anidulafungin vs. the lipid formulation of amphotericin B in high-risk patients in the early postoperative period after liver transplantation
}

\author{
S.V. Zhuravel ${ }^{\star 1}$, N.K. Kuznetsova ${ }^{1}$, I.I. Utkina', M.S. Novruzbekov', \\ P.V. Gavrilov', T.V. Chernenkaya', G.K. Ospanova ${ }^{2}$ \\ ${ }^{1}$ N.V. Sklifosovsky Research Institute for Emergency Medicine, \\ 3 Bolshaya Sukharevskaya Sq., Moscow 129090 Russia; \\ ${ }^{2}$ National Scientific Medical Center, \\ 42 Abylaykhan Ave., Nur-Sultan 020000 Kazakhstan \\ ${ }^{*}$ Correspondence to: Sergey V. Zhuravel, Dr. Med. Sci., Head of the Scientific Department for Anesthesiology, \\ Intensive and Critical Care, N.V. Sklifosovsky Research Institute for Emergency Medicine, \\ e-mail: zhsergey5@gmail.com
}

\begin{abstract}
Introduction. The development of invasive candidiasis leads to high mortality after liver transplantation. Choosing an effective prophylaxis is an important task.

The study purpose was to compare the results of invasive candidiasis prevention with anidulafungin vs. the lipid formulation of amphotericin B in high-risk patients in the early postoperative period after liver transplantation.

Material and methods. The study included 80 patients with risk factors for the development of invasive mycosis who underwent liver transplantation. Patients were divided into 2 groups. In the first group $(n=40)$, anidulafungin was prescribed for prophylaxis; in the 2 nd group $(n=40)$, the lipid complex of amphotericin $B$ was used.

Results. The most common of Candida spp. isolated in the patients of our study, as expected, was Candida albicans accounting for $31.2 \%$, significantly less than a half. Neither fungal infection breakthrough nor invasive mycosis development were reported in any patient. In group 2, renal replacement therapy was significantly more frequently used. In two cases, the amphotericin B lipid complex was canceled and the conversion to echinocandin was undertaken due to the occurrence of adverse events (chills and fever) associated, in our opinion, with the drug used.

Conclusions. 1. Patients after liver transplantation with 2 or more risk factors have absolute indications to invasive mycosis prevention. 2. Anidulafungin and the lipid formulation of amphotericin B are effective for prophylaxis and prevention of fungal infection breakthrough. 3. Anidulafungin has an advantage in safety over the lipid formulation of amphotericin $B$.
\end{abstract}

Keywords: prophylaxis of fungal infection, liver transplantation, anidulafungin, amphotericin B lipid complex

CONFLICT OF INTERests Authors declare no conflict of interest

FinANCING

The study was performed without external funding

Zhuravel SV, Kuznetsova NK, Utkina II, Novruzbekov MS, Gavrilov PV, Chernenkaya TV, et al. Comparing the results of invasive candidiasis prevention with anidulafungin vs. the lipid formulation of amphotericin B in high-risk patients in the early postoperative period after liver transplantation. Transplantologiya. The Russian Journal of Transplantation. 2020;12(2):104-111. (In Russ.). https:// doi.org/10.23873/2074-0506-2020-12-2-104-111

ИВЛ - искусственная вентиляция легких

ОПП - острое почечное повреждение

Введение

В настоящее время инфекции являются наиболее частым осложнением после трансплантации печени. Первое место, несомненно, занимают бактериальные возбудители, играющие ведущую роль в раннем послеоперационном периоде, за ними следуют вирусные и грибковые микроорганизмы $[1,2]$.

Среди грибковых инфекций Candida spp. и Aspergillus spp. являются наиболее распростра-
ПЦР - полимеразная цепная реакция

MELD - Model for End-stage Liver Disease

ненными патогенами и вызывают наиболее угрожающие жизни осложнения с высокой летальностью $[3,4]$.

Внедрение противогрибковой профилактики после трансплантации печени показало свою эффективность в снижении как частоты осложнений, так и летальности, связанной с инвазивным кандидозом при наличии одного или нескольких специфических факторов риска. Частота развития инвазивного кандидоза составляет примерно от 5 до 7\% и, учитывая крайне 
высокий процент неблагоприятных исходов при его развитии, заслуживает повышенного внимания. Инвазивный кандидоз наиболее часто развивается в течение первых 3 мес после трансплантации печени с вероятностью смертельного исхода до 70\%. По данным литературы последних лет, отмечается неуклонный рост резистентности семейства Candida spp. к фолуконазолу. Как известно, вышеуказанное средство является препаратом выбора для профилактики и лечения кандидоза на протяжении последних 15 лет. При этом проведено незначительное число исследований, посвященных выбору профилактического средства у пациентов высокого риска после трансплантации печени [5-7].

Целью нашего исследования являлось сравнение результатов профилактики анидулафунгином и липидной формой амфотерицина $B$ у пациентов высокого риска в раннем послеоперационном периоде после трансплантации печени.

\section{Материал и методы}

Мы провели сравнительное одноцентровое рандомизированное исследование профилактического применения противогрибковых лекарственных средств у пациентов высокого риска.

В исследование включили 80 реципиентов печени. Пациенты были разделены на две группы. В 1-й группе $(\mathrm{n}=40)$ с целью профилактики назначали анидулафрунгин, а во 2-й $(\mathrm{n}=40)$ применяли липидную форму амфотерицина $B$.

Критерии включения:

1) Возраст пациента 18-70 лет.

2) Наличие двух и более фракторов риска развития инвазивного кандидоза:

а) ортотопическая трансплантация печени;

б) кровопотеря более 3500 мл;

в) ретрансплантация печени;

г) релапаротомия в течение 5 сут после операции;

д) колонизация Candida spp. одного и более локусов в периоперационном периоде (ротоглотка, прямая кишка, моча, влагалище, послеоперационная рана);

е) оценка по MELD (Model for End-stage Liver Disease) более 30 баллов;

ж) применение кортикостероидов после трансплантации;

3) полное парентеральное питание более 3 сут;

и) искусственная вентиляция легких (ИВЛ) более 48 ч; к) фульминантная печеночная недостаточность.

Критерии исключения.

1. Первично нефункционирующий трансплантат.

2. Непереносимость эхинокандинов или липидной формы амфотерицина $B$.

Контролировали исходы лечения, осложнения, нежелательные явления связанные с исследуемыми препаратами, клиническую эффективность профилактической терапии и развитие инвазивной грибковой инфекции. Микробиологический мониторинг проводили перед операцией, после операции, далее 2 раза в неделю. Осуществляли посевы крови, мочи, раневого отделяемого, ротоглотки и прямой кишки с последующим анализом роста колоний на селективных средах. Кроме того, выполняли ПЦР-диагностику (ПЦР - полимеразная цепная реакция) Candida albicans, Candida glabrata, Candida parapsilosis, Candida crusei, Candida tropicalis, Candida fungi, которую осуществляли при помощи ПЦР-наборов производства ЗАО «Вектор-Бест».

\section{Статистический анализ}

Статистическую обработку данных производили с помощью программы Statistica 10. Количественные данные представлены в виде медианы и квартилей (25 и 75\%). Для сравнения результатов в группах применяли критерий Манна-Уитни. Статистическая значимость устанавливалась при значении $\mathrm{p}<0,05$.

Результаты

Всем пациентам была выполнена ортотопическая трансплантация печени. Показанием для оперативного вмешательства являлся цирроз различной этиологии. При этом у большинства пациентов как в 1-й, так и во 2-й группе показанием для трансплантации печени явился цирроз печени в исходе вирусного гепатита $C$. Медиана возраста составила 54 года $(37 ; 57)$ в 1 -й группе и 53 года $(43 ; 57)$ во 2 -й $(\mathrm{p}>0,05$, статистически незначимо) (табл. 1).

Все пациенты имели как минимум два фрактора риска развития инвазивного кандидоза в послеоперационном периоде. При этом 4 (10\%) и $3(7,5 \%)$ имели более четырех факторов риска развития инвазивного кандидоза в 1-й и во 2-й группах соответственно (табл. 2). 


\section{AGTUAL ISSUES OF TRANSPLANTHAION}

Таблица 1. Характеристика пациентов

Table 1. Patients profile

\begin{tabular}{|c|c|c|}
\hline Показатель & $\begin{array}{c}\text { Анидулафуунгин, } \\
\mathrm{n}=40\end{array}$ & $\begin{array}{c}\text { Липидный } \\
\text { комплекс } \\
\text { амфотерицина B } \\
\mathrm{n}=40\end{array}$ \\
\hline Медиана возраста & $54(37 ; 57)$ & $53(43 ; 57)$ \\
\hline $\begin{array}{l}\text { Пол: } \\
\text { Мужчин (\%) } \\
\text { Женщин (\%) }\end{array}$ & $\begin{array}{l}28(70) \\
12(30)\end{array}$ & $\begin{array}{l}22(55) \\
18(45)\end{array}$ \\
\hline \multicolumn{3}{|c|}{ Этиология цирроза, абс. (\%) } \\
\hline $\begin{array}{l}\text { Цирроз в исходе ге- } \\
\text { патита } C\end{array}$ & $13(32,5)$ & $15(37,5)$ \\
\hline $\begin{array}{l}\text { Цирроз в исходе ге- } \\
\text { патита } B\end{array}$ & $3(7,5)$ & $3(7,5)$ \\
\hline $\begin{array}{l}\text { Аутоиммунный цир- } \\
\text { роз }\end{array}$ & $3(7,5)$ & $5(12,5)$ \\
\hline $\begin{array}{l}\text { Первичный билиар- } \\
\text { ный цирроз }\end{array}$ & $1(2,5)$ & $3(7,5)$ \\
\hline $\begin{array}{l}\text { Алиментарный цир- } \\
\text { роз }\end{array}$ & $2(5)$ & $6(15)$ \\
\hline Криптогенный цирроз & $3(7,5)$ & $3(7,5)$ \\
\hline $\begin{array}{l}\text { Цирроз печени, пече- } \\
\text { ночно-клеточный рак }\end{array}$ & $5(12,5)$ & $3(7,5)$ \\
\hline Поликистоз печени & 0 & $1(2,5)$ \\
\hline $\begin{array}{l}\text { Первично склерози- } \\
\text { рующий холангит }\end{array}$ & $3(7,5)$ & 0 \\
\hline $\begin{array}{l}\text { Цирроз в исходе } \\
\text { гепатита } C+B\end{array}$ & $1(2,5)$ & 0 \\
\hline $\begin{array}{l}\text { Цирроз в исходе } \\
\text { гепатита } B+\text { +ельта }\end{array}$ & $6(15)$ & $1(2,5)$ \\
\hline
\end{tabular}

При микробиологическом и ПЦРисследованиях биологического материала грибковая колонизация двух и более локусов была выявлена у 23 пациентов (28,7\%). Эти пациенты рассматривались как группа риска по развитию инвазивного кандидоза (табл. 3).

При исследовании микробиологических посевов и ПЦР наиболее распространенным видом Candida spp. в нашем исследовании была Candida albicans, за которой следуют Candida tropicalis, Candida parapsilosis и Candida glabrata, Candida fungi, Candida crusei. Следует отметить, что нередко у пациента из разных локусов выявлялись разные микроорганизмы (табл. 4).

Медиана противогрибковой профилактики составила 7 (3; 5) сут. В группе 2 статистически значимо чаще использовалась заместительная почечная терапия. В 2 случаях осуществлены отмена липидного комплекса амфотерицина $B$ и
Таблица 2. Факторы риска развития инвазивного кандидоза

Table 2. Risk factors of invasive candidiasis development

\begin{tabular}{|c|c|c|}
\hline Показатель & $\begin{array}{l}\text { Анидулафунгин, } \\
\text { абс. (\%), n=40 }\end{array}$ & $\begin{array}{c}\text { Липидный } \\
\text { комплекс } \\
\text { амфотерицина B, } \\
\text { абс. }(\%), \mathrm{n}=40\end{array}$ \\
\hline $\begin{array}{l}\text { Трансплантация } \\
\text { печени }\end{array}$ & $40(100)$ & $40(100)$ \\
\hline $\begin{array}{l}\text { Тотальное парен- } \\
\text { теральное питание } \\
\text { более } 3 \text { сут }\end{array}$ & $40(100)$ & $40(100)$ \\
\hline $\begin{array}{l}\text { Кортикостероиды } \\
\text { после трансплан- } \\
\text { тации }\end{array}$ & $10(25)$ & $11(27,5)$ \\
\hline $\begin{array}{l}\text { Колонизация двух } \\
\text { и более локусов } \\
\text { Candida в периопе- } \\
\text { рационном периоде }\end{array}$ & $13(32,5)$ & $10(25)$ \\
\hline Ретрансплантация & $1(2,5)$ & 0 \\
\hline $\begin{array}{l}\text { Релапаротомия в } \\
\text { течение } 5 \text { сут после } \\
\text { трансплантации }\end{array}$ & $3(7,5)$ & $6(15 \%)$ \\
\hline $\begin{array}{l}\text { Кровопотеря более } \\
3500 \text { л }\end{array}$ & $2(5)$ & $1(2,5 \%)$ \\
\hline $\begin{array}{l}\text { Холодовая ишемия } \\
\text { более } 8 \text { ч }\end{array}$ & $4(10)$ & $3(7,5)$ \\
\hline ИВЛ более 48 ч & $2(5)$ & $2(5)$ \\
\hline $\begin{array}{l}\text { Оценка по MELD } \\
\text { более } 30 \text { баллов }\end{array}$ & $1(2,5)$ & $1(2,5)$ \\
\hline \multicolumn{3}{|c|}{ Количество фракторов риска, абс. (\%) } \\
\hline 1 & $40(100)$ & $40(100)$ \\
\hline 2 & $40(100)$ & $40(100)$ \\
\hline 3 & $14(35)$ & $16(40)$ \\
\hline 4 & $8(20)$ & $9(22,5)$ \\
\hline более 4 & $4(10)$ & $3(7,5)$ \\
\hline
\end{tabular}

Таблица 3. Колонизация Candida spp. у пациентов после ортотопической трансплантации печени в отделении реанимации и интенсивной терапии

Table 3. Candida spp. colonization in the intensive care unit patients after orthotropic liver transplantation

\begin{tabular}{|l|c|}
\hline Пациенты & $80(100 \%)$ \\
\hline Колонизировано, абс. (\%) & $34(42,5)$ \\
\hline Колонизация одного локуса, абс. (\%) & $11(13,75)$ \\
\hline Колонизация двух локусов, абс. (\%) & $16(20)$ \\
\hline Колонизация трех и более локусов, абс. (\%) & $7(8,7)$ \\
\hline
\end{tabular}




\section{AGTUAL ISSUES OF TRANSPLANTHTION}

Таблица 4. Лечение при колонизации Candida spp. Table 4. Candida spp. colonization treatment

\begin{tabular}{|c|c|c|}
\hline Показатель & $\begin{array}{l}\text { Анидулафунгин, } \\
\text { абс. (\%), n=40 }\end{array}$ & $\begin{array}{c}\text { Липидный комплекс } \\
\text { амфотерицина } B, \\
\text { абс. (\%), n=40 }\end{array}$ \\
\hline Всего (n) & $13(32,5)$ & $10(25)$ \\
\hline Candida albicans & $7(17,5)$ & $5(12,5)$ \\
\hline Candida glabrata & $2(5)$ & $3(7,5)$ \\
\hline $\begin{array}{l}\text { Candida } \\
\text { parapsilosis }\end{array}$ & $3(7,5)$ & $3(7,5)$ \\
\hline Candida crusei & $2(5)$ & $1(2,5)$ \\
\hline Candida tropicalis & $3(7,5)$ & $2(5)$ \\
\hline Candida fungi & $2(5)$ & $3(7,5)$ \\
\hline
\end{tabular}

конверсия на эхинокандин в связи с появлением нежелательных явлений (озноб и высокая температура), связанных, на наш взгляд, с применяемым лекарственным средством (табл. 5).

Таблица 5. Исходы и осложнения

Table 5. Outcomes and complications

\begin{tabular}{l|c|c|}
\hline \multicolumn{1}{|c|}{ Показатель } & $\begin{array}{c}\text { Анидулафунгин, } \\
\text { абс. (\%), } \mathbf{n = 4 0}\end{array}$ & $\begin{array}{c}\text { Липидный комплекс } \\
\text { амфотерицина } \mathbf{B}, \\
\text { абс. (\%), } \mathbf{n = 4 0}\end{array}$ \\
\hline $\begin{array}{l}\text { Смерть в тече- } \\
\text { ние } 30 \text { дней }\end{array}$ & $2(5)$ & $3(7,5)$ \\
\hline $\begin{array}{l}\text { Смерть во время } \\
\text { введения препа- } \\
\text { рата }\end{array}$ & 0 & 0 \\
\hline $\begin{array}{l}\text { Заместитель- } \\
\text { ная почечная } \\
\text { терапия }\end{array}$ & $4(10)$ & $10(25)^{*}$ \\
\hline $\begin{array}{l}\text { Гипертермия, } \\
\text { связанная с пре- } \\
\text { паратом }\end{array}$ & 0 & 2 \\
\hline $\begin{array}{l}\text { Отмена препа- } \\
\text { рата }\end{array}$ & 0 & 2 \\
\hline $\begin{array}{l}\text { Инвазивный кан- } \\
\text { дидоз }\end{array}$ & 0 & \\
\hline * р & \\
\hline
\end{tabular}

\section{обсуждение}

Высокий уровень летальности, связанный с инвазивным кандидозом, предполагает выбор адекватной противогрибковой терапии, которая имеет первостепенное значение у пациентов в раннем периоде после трансплантации печени. Candida albicans является наиболее распространенным микроорганизмом, при этом выявление Candida non-albicans ассоциируется с более высокой летальностью. Следует при этом отметить, что различные трансплантационные центры придерживаются собственных протоколов профилактики, которые могут значительно отличаться. В последние годы большое внимание уделяется росту устойчивости к фрлуконазолу [5]. Резистентность Candida spp. к противогрибковым препаратам представляет собой растущую проблему, общие показатели устойчивости к фрлуконазолу составляют примерно 5\% y Candida albicans. Результаты различных исследований сообщают об общих показателях устойчивости к фрлуконазолу до 57\%, что объясняется высоким уровнем выявления штаммов Candida non-albicans и предыдущей противогрибковой профилактикой. Некоторые исследователи указывают на необходимость такой профрилактики после трансплантации печени только у пациентов высокого риска [5].

Резистентность к эхинокандинам в настоящее время встречается менее чем у $2 \%$ больных при лечении Candida spp. [9].

Предотвращение развития инвазивного кандидоза предполагает всесторонний подход, включающий мониторинг грибковых патогенов, оценку фракторов риска и назначение адекватной профилактики или эмпирической терапии.

Наши результаты показывают, что анидулафругин и липидный комплекс амфотерицина B эфрфективны для профилактики кандидемии и инвазивного кандидоза у пациентов высокого риска после трансплантации печени.

Клинически значимые нежелательные явления, связанные с применением липидного комплекса амфотерицина $B$, зарегистрированные в нашем исследовании, соответствуют полученным ранее сведениям о препарате.

В нашем исследовании медиана продолжительности профилактической терапии составила 7 сут. В целом результаты исследования показывают, что реципиенты печени подвергаются риску заражения Candida, включая штаммы non-albicans. Колонизация слизистых, вовлечение ротоглотки, пищевода, прямой кишки и влагалища обычно не диагностируются как инвазивный кандидоз, но при выявлении патогенных штаммов из указанных мест следует назначение противогрибкового средства, с этим согласны все специалисты в этой области, и соответствующие рекомендации вносятся в протоколы послеоперационного ведения пациентов.

Как свидетельствуют наши результаты, следует учитывать более высокий риск разви- 
тия острого почечного повреждения (ОПП) при использовании липидного комплекса амфотерицина В. Развитие ОПП в раннем послеоперационном периоде является фрактором, влияющим на продолжительность пребывания пациента в палате интенсивной терапии, сроков госпитализации и всего лечения в целом. Отсутствие нефротоксичности у анидулафунгина является несомненным преимуществом, особенно в случае выбора лекарственного средства у пациентов высокого риска после трансплантации печени.

Эхинокандины в настоящее время - наиболее часто назначаемые противогрибковые средства в лечении кандидемии не только у пациентов с трансплантированными органами. Отметим результаты шести объединенных проспективных исследований в оценке эфрективности и безопасности применения анидулафрунгина у пациентов с кандидемией, вызванной Candida parapsilosis. Авторы приходят к выводу, что анидулафрунгин был эфрфективен для лечения кандидемии Candida parapsilosis (в исследовании были включены 70 пациентов с кандидемией, вызванной Candida parapsilosis, при этом только в 3 случаях выявлена резистентность) [10].

Анидулафунгин демонстрирует наименьшую гепатотоксичность и минимальные взаимодействия с другими лекарственными средствами при сравнении с другими группами противогрибковых препаратов. Еще раз подчеркнем, что высокий уровень резистентности к фллконазолу следует учитывать при выборе противогрибковой профилактики/эмпирической терапии. Локальные результаты мониторинга, регистрируемые фракторы риска инвазивного кандидоза способствуют разработке, динамически обновляемой стратегии профилактики, диагностики и управления инвазивными грибковыми иноекциями.

\section{Заключение}

Для предупреждения инвазивного кандидоза большое значение имеет адекватная профилактическая противогрибковая терапия у пациентов в раннем периоде после трансплантации печени, особенно при выявлении нескольких фракторов риска с сочетанием колонизации грибковыми микроорганизмами двух и более локусов.

При выборе противогрибкового лекарственного средства для профилактики следует учитывать бо́льшую безопасность анидулафрунгина в сравнении с липидным комплексом амфотерицина $B$.

Выводы

1. Для профрилактики инвазивного кандидоза имеются абсолютные показания у пациентов после трансплантации печени с двумя и более фракторами риска.

2. Анидулафрунгин и липидная фрорма амфотерицина $B$ эфрфективны для профилактики и предотвращения «прорыва» грибковой иноекции.

3. Анидулафунгин имеет преимущество перед липидной фрормой амфотерицина $B$ как более безопасное средство. 


\section{Литература / References}

1. Pappas PG, Alexander BD, Andes DR, Hadley S, Kauffman CA, Freifeld A, et al. Invasive fungal infections among organ transplant recipients: results of the Transplant-Associated Infection Surveillance Network (TRANSNET). Clin Infect Dis. 2010;50(8):1101-1111. PMID: 20218876 https://doi.org/10.1086/651262 2. Liu X, Ling Z, Li L, Ruan B. Invasive fungal infections in liver transplantation. Int $J$ Infect Dis. 2011;15(5):e298e304 PMID: 21345708 https://doi. org/10.1016/j.ijid.2011.01.005

3. Yang $\mathrm{CH}$, He XS, Chen J, Ouyang B, Zhu XF, Chen MY, et al. Fungal infection in patients after liver transplantation in years 2003 to 2012. Ann Transplant. 2012;17(4):59-63. PMID: 23274325 https://doi.org/10.12659/aot.883695

4. Eschenauer GA, Kwak EJ, Humar A, Potoski BA, Clarke LG, Shields RK, et al. Targeted versus universal antifungal prophylaxis among liver transplant recipients. Am J Transplant. 2015;15(1):180-189. PMID: 25359455 https://doi.org/10.1111/ajt.12993

5. Bassetti M, Peghin M, Carnelutti A, Righi E, Merelli M, Ansaldi F, et al. Invasive candida infections in liver transplant recipients: clinical features and risk fac- tors for mortality. Transplant Direct. 2017;3(5):e156. PMID: 28573191 https:// doi.org/10.1097/TXD.0000000000000673 6. журавель С.В., Черненькая Т.В., Баженов А.И., Оспанова Г.К. Лечение грибковых осложнений у больных после трансплантации печени. Разбор клинических случаев. Трансплантология. 2015;(3):57-64. Zhuravel SV, Chernenkaya TV, Bazhenov AI, Ospanova GK. Treatment of fungal complications in patients after liver transplantation. Discussion of clinical cases. Transplantologiya. The Russian Journal of Transplantation. 2015;(3):57-64. (In Russ.).

7. Хубутия М.Ш., Журавель С.В., Черненькая Т.В., Оспанова Г.К., Баженов А.И., Кузнецова Н.К. и др. Профилактика грибковой инфекции в раннем послеоперационном периоде после трансплантации печени. Трансплантология. 2016;(2):29-34. Khubutiya MSh, Zhuravel SV, Chernenkaya TV, Ospanova GK, Bazhenov AI, Kuznetsova NK, et al. Prevention of fungal infection in the early period after liver transplantation. Transplantologiya. The Russian Journal of Transplantation. 2016;(2):29-34.(In Russ.).

8. Климко Н.Н., Рубинчик В.Е.,
Соболь М.М., Ларионова В.Б., Тыренко В.В., Талипова Л.И. и др. Результаты проспективного многоцентрового исследования анидулофунгина - ЭРА (эраксис в Российской Федерации). Проблемы медицинской микологии. 2018;20(3):21-26. Klimko NN, Rubinchik VE, Sobol MM, Larionova VB, Tyrenko VV, Talipova LI, et al. Multicenter observational study of anidulafungin using - ERA (ERAXIS IN RUSSIA). Problems in medical mycology. 2018;20(3):21-26. (In Russ.).

9. Kullberg BJ, Vasquez J, Mootsikapun P, Nucci M, Paiva JA, Garbino J, et al. Efficacy of anidulafungin in 539 patients with invasive candidiasis: a patient-level pooled analysis of six clinical trials. J Antimicrob Chemother. 2017;72(8):2368-2377. PMID: 28459966 https://doi.org/10.1093/jac/dkx116

10. Kontoyiannis DP, Bassetti M, Nucci M, Capparella MR, Yan JL, Aram J, et al. Anidulafungin for the treatment of candidaemia caused by Candida parapsilosis: Analysis of pooled data from six prospective clinical studies. Mycoses. 2017;60(10):663-667. PMID: 28597967 https://doi.org/10.1111/ myc. 12641 


\section{АКТУАЛЬНЫЕ ВОПРОЯЫ НЛИНКЧЕНКОЙ ТРАНЯПЛАНТОЛОГИИ}

\section{AGTUAL ISSUES OF TRAMSPLANTATION}

\section{Информация об авторах}

Сергей Владимирович Журавель

д-р мед. наук, заведующий научным отделением анестезиологии, реаниматологии и интенсивной терапии ГБУЗ «НИИ скорой помощи им. Н.В. Склифосовского ДЗМ», https://orcid.org/0000-0002-9992-9260

канд. мед. наук, ведущий научный сотрудник научного отделения анестезиологии, реаниматологии и интенсивной терапии ГБУЗ «НИИ скорой помощи им. Н.В. Склифросовского ДЗМ», https://orcid.org/0000-0002-2824-1020

Кузнецова

канд. мед. наук, старший научный сотрудник научного отделения анестезиоло-

Ирина Игоревна

Уткина гии, реаниматологии и интенсивной терапии ГБУЗ «НИИ скорой помощи им. Н.В. Склифосовского ДЗМ», https://orcid.org/0000-0002-5685-4916

Мурад Сафтарович

Новрузбеков

д-р мед. наук, заведующий научным отделением трансплантации печени ГБУз «НИИ скорой помощи им. Н.В. Склифросовского ДЗМ», https://orcid.org/00000002-6362-7914

Павел Викторович

Гаврилов

младший научный сотрудник научного отделения анестезиологии, реаниматологии и интенсивной терапии ГБУЗ «НИИ скорой помощи им. Н.В. Склифосовского ДЗМ», https://orcid.org/0000-0001-9640-201X

Татьяна Витальевна

Черненькая

канд. мед. наук, заведующая научной лабораторией клинической микробиологии ГБУЗ «НИИ скорой помощи им. Н.В. Склифросовского ДЗМ», https://orcid. org/0000-0002-6167-7117

Гулбану к.

Оспанова

канд. мед. наук, старший ординатор, врач анестезиолог-реаниматолог отделения анестезиологии, реаниматологии и интенсивной терапии $\mathrm{AO}$ «Национальный научный медицинский центр», https://orcid.org/0000-0001-9673-0320

\section{Information about authors}

Sergey V. Zhuravel

Dr. Med. Sci., Head of the Scientific Department for Anesthesiology, Intensive and Critical Care, N.V. Sklifosovsky Research Institute for Emergency Medicine, https:// orcid.org/0000-0002-9992-9260

Cand. Med. Sci., Leading Research Associate of the Scientific Department for Anesthesiology, Intensive and Critical Care, N.V. Sklifosovsky Research Institute for Emergency Medicine, https://orcid.org/0000-0002-2824-1020

Cand. Med. Sci., Senior Research Associate of the Scientific Department for Anesthesiology, Intensive and Critical Care, N.V. Sklifosovsky Research Institute for Emergency Medicine, https://orcid.org/0000-0002-5685-4916

Dr. Med. Sci., Head of the Scientific Department for Liver Transplantation, N.V. Sklifosovsky Research Institute for Emergency Medicine, https://orcid. org/0000-0002-6362-7914

Junior Research Associate of the Scientific Department for Anesthesiology, Intensive and Critical Care, N.V. Sklifosovsky Research Institute for Emergency Medicine, https://orcid.org/0000-0001-9640-201X

Cand. Med. Sci., Head of the Research Laboratory for Clinical Microbiology, N.V. Sklifosovsky Research Institute for Emergency Medicine, https://orcid. org/0000-0002-6167-7117

Cand. Med. Sci., Senior Resident, Anesthesiologist-Intensivist of the Anesthesiology, Critical and Intensive Care Department, National Scientific Medical Center, https:// orcid.org/0000-0001-9673-0320 